\title{
The journey with dementia from the perspective of Bangladeshi family caregivers in England
}

Background: While Bangladeshis are three times more likely to care for dependent family members than White British (Carers UK, 2011), yet little is known about the experiences of family caregivers of people with dementia. There is a paucity of research, within the Bangladeshi community, on experiences and concerns of Bangladeshi individuals providing care for their relatives with dementia. This qualitative study explores immigrant Bangladeshi family carers' knowledge and day-to-day experiences living in England.

Methods: Semi-structured, audio-recorded interviews were conducted with six Bangladeshi family carers living in London and Portsmouth. Five interviews were conducted in English, one in Bengali later translated and transcribed to English. Data were analysed using thematic analysis. NVivo 10 software was utilised to simplify thematic analysis, including transcribing, coding, and documenting emergent themes.

Results: Three major themes emerged from the carers' interview transcripts. These were: (1) Caregiving journey into dementia; (2) Impact of dementia on family caregivers; and (3) Caregiving support and help. The current study reveals that most carers have a lack of knowledge and awareness of the symptoms of dementia. Caregivers exhibited a strong sense of familism, religious beliefs, and values which appear to increase interpersonal motive, or obligation, to provide care for their relatives with dementia at home. Family carers appeared to accept and take for granted, expectations to fulfil often-considerable caregiving roles. A scarcity of cultural and religious sensitive resources for the needs of Muslim patients saw Bangladeshi caregivers more likely to provide direct care themselves rather than receiving care from service providers. Sending a relative with dementia to a residential care home was seen as unacceptable.

Conclusions: Unlike previous studies, where South Asian carers viewed dementia as demons or God's punishments, this study found carers believed dementia was a medical condition. While previous studies reported that many South Asians were explicit in associating stigmas with dementia, all respondents in this study believed there was no stigma attached to dementia. More research is needed to further examine religious beliefs, familism, and interpersonal motives as theoretical perspectives to explain how Bangladeshi caregivers negotiate and construct their caregiving roles for their relatives with dementia. 